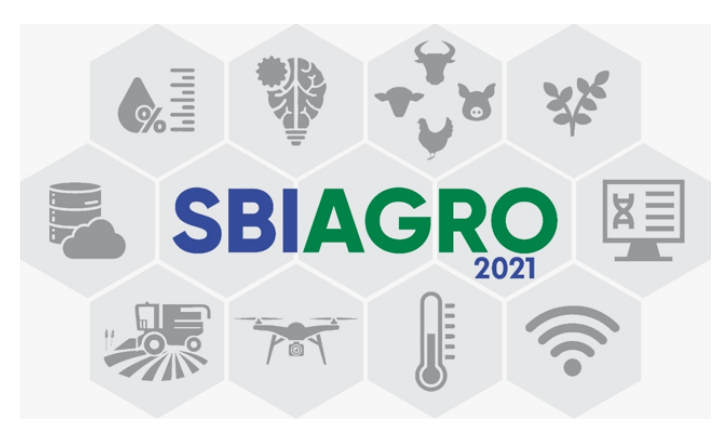

\title{
Sistemas de suporte à decisão nas fazendas inteligentes: um mapeamento sistemático
}

\author{
Jonas S. Gomes ${ }^{1}$, José Maria N. David ${ }^{1}$, Regina Braga ${ }^{1}$, Wagner Arbex ${ }^{2}$, Bryan \\ Barbosa $^{1}$, Wneiton Luiz Gomes ${ }^{2}$, Leonardo M. Gravina Fonseca ${ }^{2}$ \\ ${ }^{1}$ Departamento de Ciência da Computação, Universidade Federal de Juiz de Fora \\ (UFJF), Juiz de Fora, MG - Brazil \\ ${ }^{2}$ Empresa Brasileira de Pesquisa Agropecuária - Embrapa, Juiz de Fora, MG - Brazil

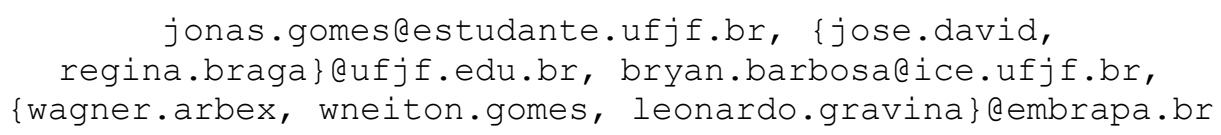

\begin{abstract}
This work presents a systematic mapping related to Decision Support Systems (DSS) in precision agriculture and livestock, in smart farming context. Through this systematic mapping, we aimed to investigate solutions that are used to support data integration from IoT sensors. The results point to the use of a knowledge base and machine learning to enrich data for decision-making. Moreover, few works exist that use semantics to enrich decision-making in the context of precision livestock.
\end{abstract}

Resumo. Este trabalho apresenta um mapeamento sistemático relacionado a Sistemas de Suporte à Decisão (SSD) na agricultura e pecuária de precisão, no contexto da agricultura inteligente. Por meio desse mapeamento sistemático, buscamos investigar soluções que são usadas para dar suporte à integração de dados de sensores IoT. Os resultados apontam para a utilização de base de conhecimento e aprendizado de máquina para enriquecimento de dados para tomada de decisão. Além disso, existem poucos trabalhos que utilizam a semântica como forma de enriquecer a tomada de decisão no contexto da pecuária de precisão.

\section{Introdução}

Nos últimos anos, a Internet das Coisas (IoT) passou a conectar diversos dispositivos e sensores, o que gerou oportunidades em vários setores, dentre eles o da agricultura e pecuária [Zhai et al 2020]. Nesse sentido, a Internet das Coisas em Saúde Animal usa biossensores e software para monitorar e manter os registros da saúde do animal. Isso gera um grande volume de dados que pode ser interpretado por sistemas inteligentes e, 
posteriormente, apoiar produtores e gestores na tomada de decisão relacionada à saúde e à produção do animal [Karthick, Sridhar e Pankajavalli 2020].

Estudos secundários recentes foram conduzidos para responder questões pertinentes a Sistemas de Suporte a Decisão no domínio da pecuária de precisão. O trabalho Villa-Henriksen et al. (2020), por exemplo, apresenta uma revisão dos padrões de interoperabilidade na pecuária de precisão que utilizam dados abertos. Esse estudo secundário discute os desafios de integrar os diversos dados gerados nas fazendas. Em [Bahlo et al. 2019] os autores revisam os problemas de rede, dispositivos físicos, aplicações e destacam o papel dos middlewares na integração de dados, uma vez que isso é um requisito fundamental para o Big Data e Inteligência Computacional. No trabalho de Zhai et al. (2020) é apresentado um survey, em que os autores ressaltam a importância da integração de dados no domínio da agricultura. Afirmam que, para a tomada de decisão, são necessários mais elementos como dados de estação meteorológica, devido à influência de temperatura e umidade na produção do animal. Nos estudos previamente apresentados, os autores abrangem a importância da integração de dados para tomada de decisão, mas não trazem uma perspectiva geral sobre como utilizar os dados coletados nas fazendas, e quais os modelos e arquiteturas de software são utilizados nos sistemas de suporte à decisão.

Dessa forma, este artigo apresenta um mapeamento sistemático cujo objetivo é responder a quatro questões de pesquisa definidas a seguir: (i) RQ1: Como integrar os dados dos Sistemas de Suporte à Decisão provenientes dos dispositivos de IoT? Através dessa pergunta, buscamos investigar o estado da arte sobre a integração de dados gerados nas fazendas pelos dispositivos IoT para dar suporte às decisões; (ii) RQ2: De que forma a semântica dos dados é tratada para integrar os Sistemas de Suporte à Decisão? Com essa pergunta buscamos identificar, nos resultados de RQ1, quais as técnicas semânticas de integração estão sendo utilizadas para enriquecer as decisões; (iii) RQ3: Quais modelos inteligentes são usados para apoiar os Sistemas de Suporte à Decisão na agricultura e pecuária de precisão? Dada a crescente utilização da inteligência computacional, apontada nos trabalhos secundários, essa questão visa explorar os modelos, técnicas e algoritmos inteligentes que estão sendo utilizados na smart farming; (iv) RQ4: Como a computação ubíqua está sendo aplicada na agricultura e pecuária de precisão? Considerando que a Internet das Coisas vem sendo amplamente utilizada na Agricultura 4.0, como mostra os estudos secundários, o intuito dessa questão é analisar como as soluções utilizadas contribuem para o avanço da computação ubíqua nas fazendas, seja através do monitoramento através de sensores, no uso do celular, de "drones" ou de dispositivos inteligentes.

Através de um mapeamento sistemático, nosso foco é elencar as técnicas e abordagens que são usadas para dar suporte à integração de dados nas fazendas para que os Sistemas de Suporte à Decisão (SSD) possam apoiar as decisões no contexto da agricultura e pecuária de precisão. Este artigo está organizado da seguinte forma: a Seção 2 discute a metodologia da pesquisa. Na Seção 3 apresentamos o processo de coleta de dados. Em seguida, discutimos os resultados obtidos e, na Seção 5, as apresentamos as considerações finais. 


\section{Metodologia de Pesquisa}

A metodologia utilizada neste trabalho segue as orientações propostas por Kitchenham (2004). Durante a condução do mapeamento, foi utilizado a ferramenta de suporte denominada Parsifal ${ }^{1}$. O detalhamento completo do mapeamento, como questões de qualidade e critérios de inclusão e exclusão, está disponível online ${ }^{2}$.

\subsection{Etapas de Pesquisa}

De acordo com as recomendações de Kitchenham (2004), a pesquisa foi dividida em três partes, planejamento, condução e relatório. Durante o planejamento foram identificadas as necessidades para revisão, as questões de pesquisa que deveriam ser respondidas e o protocolo a ser seguido. Feito isso, iniciou-se o processo de condução, através do qual identificamos e selecionamos os estudos, e executamos as técnicas de backward e forward snowballing, conforme a técnica de busca híbrida [Mourão et al. 2017]. A técnica de busca híbrida consiste em executar a string de busca um uma base que indexa outras bases de dados, como a Scopus. Em seguida, executar a técnica de backward e forward snowballing nos artigos retornados pela string. Após isso, foi realizada uma extração e síntese dos dados obtidos para uma análise de qualidade, e apresentamos os resultados.

\subsection{Termos de Pesquisa}

Os termos de pesquisa foram definidos considerando a População, Intervenção, Comparação, Resultado e Contexto (PICOC) [Kitchenham 2007], a fim de identificar palavras-chave, sinônimos e construir a string de busca com base na combinação dos termos-chave. Comparação não é relevante neste trabalho, uma vez que este mapeamento é um estudo exploratório. Para selecionar os artigos a serem analisados a fim de responder às questões de pesquisa, alguns critérios foram definidos. Os critérios de inclusão visaram adicionar aqueles trabalhos que apresentam arquiteturas, integração de dados e modelos inteligentes para apoiar as decisões na agricultura e pecuária de precisão, e estudos em inglês. Os critérios de exclusão adotados foram: (i) capítulos de livro, (ii) estudos publicados apenas como resumo, (iii) estudos cuja versão é mais antiga em relação a outro estudo já considerado, (iv) artigos que não estivessem em inglês, (v) trabalhos que não apresenta um resumo e (v) as palavras-chave não estão presentes no resumo. Mais detalhes do processo de seleção podem ser vistos em: (https://files.fm/fl65d7wxg3p).

\subsection{String de Busca}

A busca foi feita na base de dados eletrônica da Scopus, uma vez que ela indexa diversas outras bases, possibilitando a execução de apenas uma string de busca [Mourão et al. 2017]. Um conjunto de estudos primários (artigos de controle) [Villa-Henriksen et al. 2020] e [Helfer et al. 2019] foi definido, como sugerido em [Budgen et al. 2008], para a precisão da string de busca nas bases de dados selecionadas e se a pesquisa recuperou estudos relevantes. As palavras-chave desses artigos foram analisadas para encontrar novos termos relevantes a serem incluídos como parte da string de busca. Especialistas

\footnotetext{
${ }^{1} \mathrm{https}: / /$ parsif.al/

${ }^{2}$ https://files.fm/f/65d7wxg3p
} 
na área de Sistemas de Suporte à Decisão também participaram da revisão dos termos da string. A string de busca final foi descrita da seguinte maneira:

\section{("internet of things" OR iot) AND (dss OR “decision support system") AND (agriculture OR livestock).}

\section{Coleta de dados}

O primeiro passo nesta fase foi executar a string de busca, considerando as fontes selecionadas. Posteriormente, foi realizado o processo de seleção dos estudos, ilustrado na Figura 1, que compreendeu 4 etapas principais.

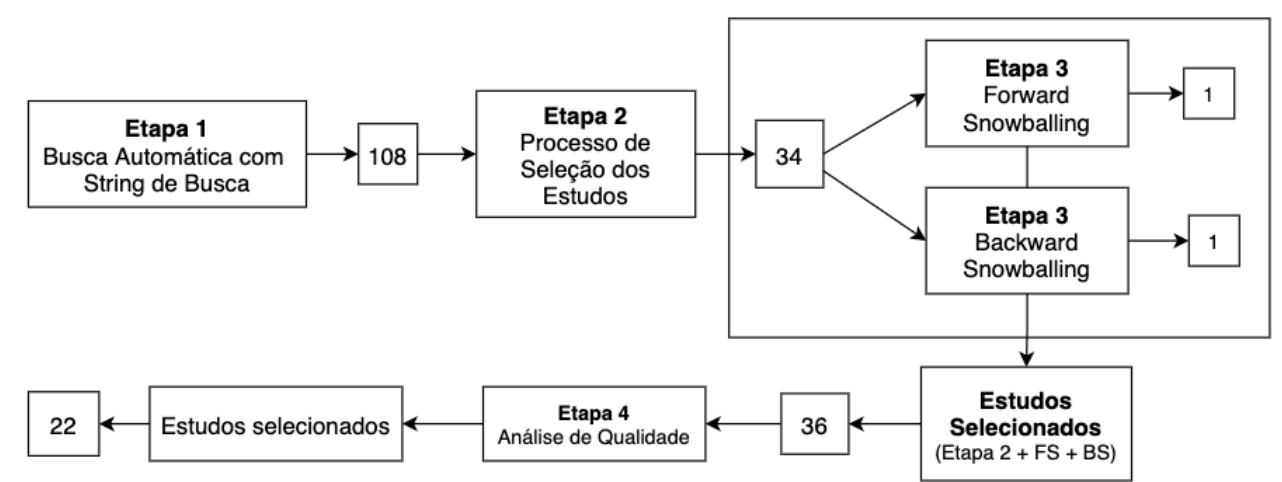

Figura 1: Processo de coleta de dados [Mourão et al. 2017]

Na Etapa 1 os resultados da busca nas bases de dados foram mesclados no Parsifal, e os trabalhos duplicados foram removidos, totalizando 108 artigos. A Etapa 2 envolveu a análise do título e do resumo considerando os critérios de inclusão/exclusão. Aqueles artigos que não abordavam Sistemas de Suporte à Decisão e IoT foram excluídos. Foram lidas as seções de Introdução, Referencial Teórico e Conclusão dos artigos. Como resultado, 74 artigos (68\%) foram excluídos e 34 (32\%) incluídos.

De acordo com as diretrizes propostas em [Kitchenham 2004], os pesquisadores devem desenvolver listas de verificação de qualidade para avaliar os estudos individuais. Durante a Etapa 3, aplicou-se o backward e forward snowballing [Webster e Watson 2002] aos 34 artigos selecionados resultando em mais 1 artigo pelo backward snowballing e 1 pelo forward snowballing.

$\mathrm{Na}$ Etapa 4, os trabalhos foram lidos na íntegra e sua qualidade foi avaliada por meio da lista de perguntas de avaliação de qualidade, que foi personalizada para o contexto deste mapeamento. Nesta etapa também executamos a extração de dados, verificando qual abordagem as soluções utilizaram para apoiar Sistemas de Suporte à Decisão e sua aplicação na agricultura ou pecuária de precisão. Esta seleção foi realizada por especialistas nas áreas de Sistemas de Suporte à Decisão. Ao final da etapa 4, dos 36 artigos, 22 (64\%) foram incluídos. Esta redução nos estudos pode ser justificada pelos seguintes aspectos: (i) ausência de propostas de soluções para apoiar a tomada de decisão; (ii) muitos estudos discutiram a relevância da integração no contexto de IoT e tomada de decisão, sem introduzir uma solução para sua realização; e (iii) a pontuação na lista de verificação de avaliação de qualidade. Uma vez mapeados os trabalhos, foi necessária uma análise mais profunda a fim de identificar, avaliar e interpretar os estudos selecionados para responder às questões de pesquisa do mapeamento sistemático. 


\section{Resultados}

De acordo com os resultados encontrados no mapeamento é possível observar que a utilização de web semântica e bases de conhecimento semântico estão sendo amplamente utilizados. A aplicação dessas soluções varia desde a irrigação de plantações e fertilização do solo, ao cuidado e manejo dos animais para que possam aumentar sua produção. Cerca de 11 artigos (50\%) selecionados para o mapeamento abordam alguma técnica de web semântica, seja utilização de ontologias para inferir novos conhecimentos e marcações semânticas para análise automática de algoritmos inteligentes. Os demais artigos variam sua abordagem em relação à utilização de inteligência computacional. Redes neurais, Support Vector Machine (SVM), aprendizado de máquina e aprendizado profundo também são algumas das técnicas utilizadas. A Tabela 1 sintetiza os achados do mapeamento.

Tabela 1: Resumo das descobertas do relatório de mapeamento.

\begin{tabular}{|c|l|}
\hline Artigos & \multicolumn{1}{|c|}{ Aplicação } \\
\hline S1 & Consciência de Contexto e Middleware \\
\hline S2 & Aprendizado de máquina \\
\hline S3, S18 & Ontologias e Web Semântica \\
\hline S4, S5 & Inteligência computacional com árvore de decisão \\
\hline S6, S15, S17, S22 & Web Semântica \\
\hline S7, S8, S12, S14, S20 & Base de conhecimento semântica \\
\hline S9 & Inteligência computacional com redes de Petri \\
\hline S10, S11, S19 & Análise Estatística \\
\hline S13 & Aprendizado profundo \\
\hline S5, S16 & Inteligência computacional com SVM \\
\hline S21 & Inteligência computacional com Redes neurais \\
\hline
\end{tabular}

RQ1: Como integrar os dados dos Sistemas de Suporte à Decisão provenientes dos dispositivos de IoT? As pesquisas conduzidas em [S6, S15, S17, S19, S22] demonstram a utilização de uma rede de sensores sem fio (WSN) para integrar todos os sensores IoT do campo, a fim de fornecer o maior conjunto de dados para os sistemas de suporte à decisão. Em [S15] é proposto um protótipo de aplicativo móvel integrado com a rede de sensores sem fio, capaz de emitir alertas via SMS para que os produtores possam tomar decisões rapidamente sobre a situação do solo. Kamath et al. [S17] descrevem a implementação de uma rede de sensores sem fio para monitorar culturas de arroz com captura de imagem. Os autores fizeram a integração do sistema com o RabbitMQ [S17], um serviço de mensageria capaz de persistir os dados dos sensores. Em [S19] são propostos um modelo conceitual e projeto de sistema para apoio à decisão na smart farming. $\mathrm{O}$ trabalho utiliza um middleware para dar suporte à comunicação e à interoperabilidade de dispositivos inteligentes, a detecção e caracterização de eventos em tempo real e a coleta de eventos para análise de big data. Khanna e Kaur [S22] utilizam 
uma rede de sensores sem fio integrados com servidores em nuvem. Dessa forma, os produtores podem monitorar diferentes informações das plantações, como temperatura e umidade do solo, e intervir nos locais físicos para equilibrar o ambiente. A pesquisa proposta em [S3] descreve o Agri-IoT, um framework baseado em IoT capaz de integrar via Global Sensor Network (GSN) vários fluxos de dados, fornecendo um processamento semântico completo.

RQ2: De que forma a semântica dos dados é tratada para integrar os Sistemas de Suporte à Decisão? Os trabalhos [S1, S3, S18] propõem o uso de uma camada que trata da semântica para interpretar os dados de dispositivos IoT, e [S1] ainda agrega dados de contexto como temperatura, pressão, velocidade do vento, umidade, raios $\mathrm{UV}$, entre outros dados que podem influenciar o comportamento dos animais, das colheitas e plantações. Uma vez que esses dados são levados em consideração, é possível que os sistemas indiquem decisões mais apropriadas no manejo das fazendas. Kamilaris et al. [S3] exploram como a integração semântica de informações de várias fontes, tais como: sensores, mídia social, fazendas conectadas, alertas governamentais, regulamentações, entre outras, pode aumentar a produtividade em smart farming. Já em [S5] os autores propõem o uso de web semântica para enriquecer os dados gerados pelos dispositivos IoT e, em seguida, aplicar algoritmos de aprendizado de máquina para otimizar a fertilização do solo. Com isso, a evolução da agropecuária tradicional para agricultura e pecuária de precisão, sistemas conectados e baseados em conhecimento semântico, têm transformado o ambiente físico e tecnológico das propriedades rurais. Os proprietários, produtores e veterinários ganham um novo papel nesse contexto: enriquecer os sistemas através de uma base de conhecimento compartilhado, de tal forma que as decisões sejam cada vez mais eficientes.

RQ3: Quais modelos inteligentes são usados para apoiar os Sistemas de Suporte à Decisão na agricultura e pecuária de precisão? Os artigos [S2, S4, S5, S9, S13, S16, S21] apresentaram soluções de inteligência computacional variando entre as técnicas de SVM, árvore de decisão, redes de Petri e redes neurais. Através da coleta dos dados gerados pelos dispositivos IoT no campo, é possível utilizar técnicas de aprendizado de máquina para predição de eventos como colheita, irrigação e fertilização do solo [S5, 16, S21]. Todos esses trabalhos apresentaram a computação na nuvem como componente importante da solução, uma vez que a comunicação entre sensores, sistemas e repositórios se dá através da web. Os trabalhos [S7, S8, S11, S12, S14, S20] utilizam bases de conhecimento com regras específicas do domínio da agricultura. Essas regras servem de bases tanto para os sistemas indicarem decisões, quanto para que os algoritmos inteligentes tenham os parâmetros corretos para realizar uma predição confiável. Nesses estudos é possível notar variações de abordagem, tais como a automatização de decisões através da predição de eventos indicados pelos algoritmos de aprendizado de máquina [S2, S9, S13, S5, S16, S21], até conhecimentos que podem ser inferidos utilizando web semântica [S6, S15, S17, S22]. Adicionalmente, camadas que tratam da semântica são utilizadas para refinar a análise de aprendizado de máquina para apoiar Sistemas de Suporte à Decisão, tornando cada vez mais ricas e confiáveis as decisões.

RQ4: Como a computação ubíqua está sendo aplicada na pecuária de precisão? Após a análise dos estudos, foi possível perceber como a computação ubíqua está presente nas fazendas. Em [S10] os autores propõem duas aplicações móveis integradas, uma para o produtor e outra para o vendedor de produtos agrícolas. $\mathrm{Na}$ pesquisa, o produtor pode monitorar e enviar os dados de sua plantação para o vendedor, 
que por sua vez, pode indicar o melhor fertilizante, pesticida e quantidade de água necessária. Alguns trabalhos, como [S3], demonstram a utilização de "drones" para coleta de imagens de ambientes agrícolas. Isto pode ser estendido para localizar animais no pasto, monitorar suas atividades de movimentação e detectar animais doentes. Dessa maneira, os "drones" podem agilizar o processo de decisão fornecendo informações em tempo real. A criação de uma rede de sensores sem fio também contribui para que um sistema possa gerenciar mais de uma fazenda geograficamente distribuída, podendo indicar diferentes decisões simultaneamente de acordo com os dados coletados pelos sensores.

\subsection{Ameaças à Validade}

Este mapeamento teve como objetivo explorar questões relacionadas aos Sistemas de Suporte à Decisão no contexto da agricultura e pecuária de precisão. Entretanto, algumas ameaças à validade podem ser ressaltadas. Em relação à string de busca, mesmo utilizando um processo de revisão dos termos é possível que algum termo não tenha sido considerado para o contexto de agricultura de precisão. Para mitigar essa ameaça, utilizamos a técnica de snowballing.

A utilização da base Scopus também representa uma ameaça à validade. Outras bases não indexadas pela base utilizada podem conter pesquisas relevantes para este mapeamento. Para mitigar essa ameaça caberia explorar outras bases específicas, sobretudo voltadas para as pesquisas no agronegócio. Entretanto, consideramos que as bases utilizadas nesta pesquisa foram capazes de oferecer uma ampla visão geral do estado da arte das pesquisas relacionadas à Sistemas de Suporte à Decisão voltadas para o Agronegócio.

\section{Conclusão e Trabalhos Futuros}

Este artigo apresentou um mapeamento sistemático para identificar, classificar e analisar soluções computacionais em Sistemas de Suporte à Decisão na agricultura e pecuária de precisão. Inicialmente, 108 artigos foram identificados pela fonte selecionada, os quais foram filtrados, e isso resultou em 34 artigos. Após essa seleção, foi executada a técnica de snowballing agregando mais 2 novos artigos, totalizando 36 trabalhos. Após a análise de qualidade, o resultado totalizou 22 artigos.

Como contribuição, este mapeamento oferece oportunidades de pesquisas a partir da busca pelo estado da arte das pesquisas em SSD. Ao avançar no conhecimento sobre o tema, apresentamos oportunidades e técnicas de integração utilizadas nos trabalhos, demonstrando a importância de se utilizar dados de sensores IoT para apoiar as decisões. Como resultado, decisões complexas voltadas para o agronegócio poderão ser enriquecidas com informações existentes em outros sistemas utilizados pelas propriedades rurais. Para trabalhos futuros, outras questões de pesquisa podem ser exploradas sobretudo no que diz respeito à utilização da integração entre sistemas e dados gerados em dispositivos da IoT, para o agronegócio. Adicionalmente, é importante desenvolver pesquisas que tratam da proveniência da decisão, levando em consideração o contexto dos dados de IoT, para enriquecer e melhorar os SSD na agropecuária. 


\section{Agradecimentos}

Gostaríamos de agradecer aos pesquisadores que participaram da avaliação e expressar nossos agradecimentos ao Centro Nacional de Pesquisa da Pecuária de Leite (Embrapa Gado de Leite) da Empresa Brasileira de Pesquisa Agropecuária (Embrapa). Este trabalho foi parcialmente financiado pela UFJF / Brasil, CNPq / Brasil (bolsa 311595 / 2019-7) e FAPEMIG / Brasil (bolsa APQ-02685-17), (bolsa APQ-02194-18). Este estudo também foi realizado com apoio da Coordenação de Aperfeiçoamento de Pessoal de Nível Superior - Brasil (CAPES) - Código de Financiamento 001.

\section{Referências}

Bahlo, C., Dahlhaus, P., Thompson, H., Trotter, M. (2019) "The role of interoperable data standards in precision livestock farming in extensive livestock systems: A review". Computers and electronics in agriculture. Vol. 156, pp. 459-466, doi: 10.1016/j.compag.2018.12.007.

Budgen, D., Turner, M., Brereton P., Kitchenham, B. (2008) "Using mapping studies in software engineering," in Proceedings of PPIG.

Helfer, G. A., Barbosa, J. L., Costa, A. B. D., Martini, B. G., Santos, R. D. (2019) "A model for productivity and soil fertility prediction oriented to ubiquitous agriculture". In Proceedings of the 25th Brazillian Symposium on Multimedia and the Web, pp. 489-492, doi: 10.1145/3323503.3360637.

Karthick, G. S., Sridhar, M., Pankajavalli, P. B. (2020) "Internet of things in animal healthcare (IoTAH): review of recent advancements in architecture, sensing technologies and real-time monitoring”. SN Computer Science, 1(5).

Kitchenham B. (2004) "Procedures for performing systematic review". Vol. 33, Keele University, Keele, UK.

Kitchenham, B., Charters S. (2007) "Guidelines for performing Systematic Literature Reviews in Software Engineering". Keele University and University of Durham, EBSE Technical Report Version 2.3.

Mourão E., Kalinowski M., Murta L., Mendes E., Wohlin C. (2017) "Investigating the Use of a Hybrid Search Strategy for Systematic Reviews," 2017 ACM/IEEE International Symposium on Empirical Software Engineering and Measurement (ESEM), pp. 193-198, doi: 10.1109/ESEM.2017.30.

Villa-Henriksen, A., Edwards, G. T., Pesonen, L. A., Green, O., Sørensen, C. A. G. (2020) "Internet of Things in arable farming: Implementation, applications, challenges and potential". Biosystems Engineering, Volume 191, pp. 60-84, doi: 10.1016/j.biosystemseng.2019.12.013.

Webster, J., Watson, R. T. (2002). "Analyzing the past to prepare for the future: Writing a literature review". MIS quarterly, Vol. 26, No.2, pp. xiii-xxiii, doi: http://www.jstor.org/stable/413231.

Zhai Z., Martínez F. J., Beltran V., Martínez L. N. (2020) “Decision support systems for agriculture 4.0: Survey and challenges". Computers and Electronics in Agriculture, Volume 170, March 2020, 105256, doi: 10.1016/j.compag.2020.105256. 


\section{ESTUDOS SELECIONADOS}

[S1] Symeonaki E., Arvanitis K., Piromalis D. (2020) "A Context-Aware Middleware Cloud Approach for Integrating Precision Farming Facilities into the IoT toward Agriculture 4.0". Applied Sciences.10(3), 813; https://doi.org/10.3390/app10030813.

[S2] Kakamoukas, G. et al., (2019) "A Multi-collective, IoT-enabled, Adaptive Smart Farming Architecture," 2019 IEEE International Conference on Imaging Systems and Techniques (IST), pp. 1-6, doi: 10.1109/IST48021.2019.9010236.

[S3] Kamilaris, A., Gao, F., Prenafeta-Boldu, F. X., Ali, M. I. (2016) "Agri-IoT: A semantic framework for Internet of Things-enabled smart farming applications," 2016 IEEE 3rd World Forum on Internet of Things (WF-IoT), pp. 442-447, doi: 10.1109/WF-IoT.2016.7845467.

[S4] Rezk, N. G., Hemdan, E. E., Attia, A., El-Sayed, A., Elrashidy, M.A. (2021) "An efficient IoT based smart farming system using machine learning algorithms". Multimedia Tools and Applications, vol. 80, pp. 773-797.

[S5] Sowmya B. J., Krishna Chaitanya S., Seema S., K.G. Srinivasa, (2020) "Data Analytic Techniques for Developing Decision Support System on Agrometeorological Parameters for Farmers," International Journal of Cognitive Informatics and Natural Intelligence (IJCINI), IGI Global, vol. 14(2), pp. 92-107. doi: 10.4018/IJCINI.2020040106.

[S6] Visconti P., de Fazio R., Velázquez R., Del-Valle-Soto C., Giannoccaro N. I. (2020) "Development of Sensors-Based Agri-Food Traceability System Remotely Managed by a Software Platform for Optimized Farm Management”. Sensors. 20(13), 3632. doi: $10.3390 / \mathrm{s} 20133632$.

[S7] Mikhaylenko, I. M., Timoshin, V. N. (2019). "Expert strategic management systems in precision farming". In Journal of Physics: Conference Series. IOP Publishing. doi: 10.1088/1742-6596/1419/1/012030.

[S8] Rekha, P., Rangan, V. P., Ramesh, M. V., Nibi, K. V. (2017) "High yield groundnut agronomy: An IoT based precision farming framework," 2017 IEEE Global Humanitarian Technology Conference (GHTC). pp. 1-5, doi: 10.1109/GHTC.2017.8239287.

[S9] Borisenko, I., Gangur, M.. Kobylko, A., Romanov, D., Smirnova, O., (2019) "Information support system for management decisions in the agricultural sector", 2019 International Scientific Conference on Agricultural Machinery Industry, Volume 403, Issue 1. doi: 10.1088/1755-1315/403/1/012083.

[S10] Dabre, K. R., Lopes, H. R., D'monte, S. S. (2018) "Intelligent Decision Support System for Smart Agriculture," 2018 International Conference on Smart City and Emerging Technology. pp. 1-6, doi: 10.1109/ICSCET.2018.8537275.

[S11] Yusianto, R. R., Marimin, Suprihatin, Hardjomidjojo, H. (2020) "Intelligent Spatial Decision Support System Concept in the Potato Agro-Industry Supply Chain," 2020 International Conference on Computer Science and Its Application in Agriculture (ICOSICA). pp. 1-7, doi: 10.1109/ICOSICA49951.2020.9243233.

[S12] Loret, N., Affinito, A., Bonanomi, G. (2020). "Introducing Evja-Rugged Intelligent Support System for precision farming”. ACTA IMEKO, 9(2), pp. $83-88$. 
[S13] Kale, A. P., Sonavane, S. P. (2019) "IoT based Smart Farming: Feature subset selection for optimized high-dimensional data using improved GA based approach for ELM". Computers and Electronics in Agriculture, Volume 161, pp. 225-232. doi: 10.1016/j.compag.2018.04.027

[S14] Zhang X., Zhang J., Li L., Zhang Y., Yang G. (2017) "Monitoring Citrus Soil Moisture and Nutrients Using an IoT Based System”. Sensors (Basel). 2017;17(3):447. doi:10.3390/s17030447.

[S15] Karim, F., Karim, F., Ali frihida, (2017) "Monitoring system using web of things in precision agriculture". Procedia Computer Science, Volume 110, pp. 402-409. doi: 10.1016/j.procs.2017.06.083.

[S16] Tripathy, P. K., Tripathy, A. K., Agarwal, A., Mohanty, S. P. (2021) "MyGreen: An IoT-Enabled Smart Greenhouse for Sustainable Agriculture," IEEE Consumer Electronics Magazine. vol. 10, no. 4, pp. 57-62, 1 July 2021, doi: 10.1109/MCE.2021.3055930.

[S17] Kamath, R., Balachandra, M., Prabhu, S. (2019) "Raspberry Pi as Visual Sensor Nodes in Precision Agriculture: A Study," in IEEE Access, vol. 7, pp. 45110-45122, doi: 10.1109/ACCESS.2019.2908846.

[S18] Rotondi, D., Straniero, L., Saltarella, M., Balducci, F., Impedovo, D., Pirlo, G. (2019) "Semantics for Wastewater Reuse in Agriculture*," 2019 IEEE International Conference on Systems, Man and Cybernetics (SMC), pp. 598-603, doi: 10.1109/SMC.2019.8913949.

[S19] Suakanto, S., Engel, V. J. L., Hutagalung, M., Angela, D. (2016) "Sensor networks data acquisition and task management for decision support of smart farming," 2016 International Conference on Information Technology Systems and Innovation (ICITSI). pp. 1-5, doi: 10.1109/ICITSI.2016.7858233.

[S20] Sakthi, U., Rose, J. D. (2020) "Smart Agricultural Knowledge Discovery System using IoT Technology and Fog Computing," 2020 Third International Conference on Smart Systems and Inventive Technology (ICSSIT). pp. 48-53, doi: 10.1109/ICSSIT48917.2020.9214102.

[S21] Balan T., Dumitru C., Dudnik G., Alessi E., Lesecq S., Correvon M., Passaniti F., Licciardello A. (2020) "Smart Multi-Sensor Platform for Analytics and Social Decision Support in Agriculture”. Sensors. 20(15), 4127. doi: 10.3390/s20154127.

[S22] Khanna, A., Kaur, S. (2020) "Wireless Sensor and Actuator Network (s) and its significant impact on Agricultural domain".

2020 Sixth International Conference on Parallel, Distributed and Grid Computing (PDGC), pp. 384-389, doi: 10.1109/PDGC50313.2020.9315822. 